\title{
„Die Psychiatrie nimmt sich Zeit ...“ Warum Psychiater*in werden? - Eine qualitative Studie
}

\author{
“Psychiatry Takes its Time... " Why Does One Become \\ a Psychiatrist? - A Qualitative Study
}

\section{(ㄷ)(1) $(-5)$}

\author{
Autoren \\ Annemarie Unger ${ }^{1}$, Rebecca Jahn ${ }^{1}$, Anna Höflich ${ }^{1}$, Maria Gruber ${ }^{1,2}$
}

Schlüsselwörter

Psychiatrie, Wahl des Karrierefachs, Einstellung

Keywords

psychiatry, career choice, attitude

Online-Publikation 20.5.2021

Bibliografie

Psychiat Prax 2022; 49: 198-204

DOI 10.1055/a-1472-5169

ISSN 0303-4259

(C) 2021. The Author(s).

This is an open access article published by Thieme under the terms of the Creative Commons Attribution-NonDerivative-NonCommercial License, permitting copying and reproduction so long as the original work is given appropriate credit. Contents may not be used for commercial purposes, or adapted, remixed, transformed or

built upon. (https://creativecommons.org/licenses/by-nc-nd/4.0/)

Georg Thieme Verlag KG, Rüdigerstraße 14,

70469 Stuttgart, Germany

Korrespondenzadresse

Maria Gruber, Universitätsklinik für Psychoanalyse und

Psychotherapie, Medizinische Universität Wien,

Währinger Gürtel 18-12, 1090 Wien, Österreich

maria.gruber@meduniwien.ac.at

\# Zusätzliches Material finden Sie unter:

https://doi.org/10.1055/a-1472-5169

\section{ZUSAMMENFASSUNG}

Ziel Identifikation von intrinsischen Motivatoren, die bei der Berufswahl Psychiatrie eine Rolle spielen.

Methodik 14 leitfadengestützte Tiefeninterviews wurden mithilfe von Codierungsrahmen systematisch ausgewertet. Ergebnisse Positive Aspekte waren der zwischenmenschliche Fokus und der ganzheitliche Ansatz. Negative Dimensionen waren das schlechte Image unter Kolleg*innen, Vorurteile und Stigma. Die Entstigmatisierung der Psychiatrie und die Gleichstellung psychiatrischer mit somatischen Aspekten in Fallberichten wurden als Möglichkeiten vorgeschlagen, das Interesse am Fach Psychiatrie zu erhöhen.

Schlussfolgerung Um die Beliebtheit der Psychiatrie bei Mediziner*innen zu steigern, bedarf es Maßnahmen zur Bekämpfung von mit Psychiatrie verbundenem Stigma im Gesundheitswesen. Im Rahmen der medizinischen Lehre müssen psychiatrische Störungen, welche durch ihre hohe Prävalenz alle medizinischen Disziplinen betreffen, entsprechende Gewichtung erhalten.

\section{ABSTRACT}

Objective Identification of intrinsic motivators involved in choosing psychiatry as a career path.

Methods 14 qualitative in-depth interviews were analysed systematically using coding frames.

Results Positive findings were the interpersonal focus and the holistic approach of psychiatry. Negative dimensions were the unfavorable image among colleagues, the lack of precision, prejudices and stigmatization. To interest more medical students, cases should be presented weighing psychiatric aspects as equally important to other medical aspects.

Conclusions To increase the popularity of psychiatry, the prejudices inherent in the medical system need urgent addressing. Teaching should be conducted in case presentations. Psychiatric conditions, which are highly prevalent across all medical fields, need to be adequately represented. 


\section{Hintergrund}

Der steigende Bedarf an psychiatrischer Versorgung in Österreich sieht eine hohe Anzahl von Fachärzt*innen für Psychiatrie vor, welche die notwendigen Behandlungen gewährleisten können. Allerdings scheint sich die Situation der fachärztlichen Versorgung aufgrund einer alternden Ärzt*innenschaft, einem Ärzt*innenmangel im Allgemeinen und von Psychiater*innen im Besonderen in Zukunft zu verschärfen [1].

Eine 2011 veröffentlichte Studie im Auftrag der Österreichischen Gesellschaft für Neuropsychopharmakologie und Biologische Psychiatrie bestätigt, dass ab 2017 der Bedarf an Psychiater*innen das Angebot übersteigt und die Versorgungslücke bis 2030 auf rund 600 fehlende Vollzeit-Psychiater*innen ansteigen wird [2].

Nur wenige Medizinstudent*innen interessieren sich für Psychiatrie als Berufsweg [3]. International wählen nur 3,5-4,9\% dieses Fachgebiet [4-6], sodass die zukünftige Rolle der Psychiater*innen in der Versorgung psychisch Kranker ungewiss ist [7]. Dementsprechend waren die letzten Jahre in Österreich durch eine fehlende Nachbesetzung offener Fachärzt*innenstellen an zahlreichen großen psychiatrischen Kliniken und Kassenplanstellen im ländlichen Raum gekennzeichnet [8].

Bisherige Studien zum Interesse an einer Karriere in der Psychiatrie sind zahlreich, die meisten wurden mit standardisierten Fragebögen durchgeführt, die Medizinstudent*innen und fertigen Mediziner*innen vorgegeben wurden, und beinhalten keine qualitativen Daten [9-13]. Die bisherigen Erhebungen konnten zeigen, dass sich die Einstellung der Student*innen gegenüber Psychiatrie und psychiatrischen Patient*innen durch die Teilnahme an einem klinischen Praktikum signifikant verbesserte. Was sich jedoch auch zeigte war ein deutlich negatives Bild von Psychiater*innen durch Kolleg*innen sowie die Ansicht, dass psychiatrische Patient*innen als besonders anstrengend gelten.

Obwohl in all den genannten Instrumenten verschiedene Aspekte der Psychiatrie beleuchtet werden, ist der Großteil der Aussagen negativ formuliert, was als problematisch gesehen werden muss, da positive Aspekte unzureichend erfasst werden und somit ein differenziertes Bild nicht möglich ist. Außerdem werden die Antworten auf einer 4-stelligen Likert-Skala ohne neutrale Auswahlmöglichkeit angeboten, sodass indifferente Antworten vermieden werden. Ein weiterer Kritikpunkt bezieht sich darauf, dass die vorhandenen Fragebögen die tatsächlichen Erfahrungen und Meinungen von Medizinstudent*innen oder klinisch tätigen Psychiater*innen nicht in ausreichender Tiefe berücksichtigen. Während das subjektive Bild des Faches Psychiatrie und die persönlichen Erfahrungen der Einzelnen in der Praxis letztlich ausschlaggebend für das weitere berufliche Interesse an diesem Fachgebiet sind, geschieht die Berücksichtigung dieser individuellen Einstellungen in der Forschung noch relativ selten. Insbesondere qualitative Studien zu diesem Thema wurden bisher kaum durchgeführt und publiziert, obwohl sie besonders geeignet sind, die Rekonstruktion subjektiver Erfahrungen und Ansichten zu ermöglichen.

Ziel dieser Studie ist es daher, Medizinstudent*innen mit wenig klinischer Erfahrung im Fach Psychiatrie sowie Ärzt*in- nen in der Fachärzt*innenausbildung für Psychiatrie und Fachärzt*innen zu ihrem subjektiven Bild von der Psychiatrie und ihrer persönlichen Motivation, sich für oder gegen eine Berufsausbildung in diesem Bereich zu entscheiden, zu befragen. Durch die qualitative Datenerhebung sollen neue Impulse für die Forschung und die Politik entstehen, um mehr Menschen für eine Berufsausbildung in der Psychiatrie zu interessieren.

\section{Methodik}

\section{Studiendesign und Durchführung}

Das Konzept dieser Studie wurde bei der Ethikkommission der Medizinischen Universität Wien zur Prüfung eingereicht und befürwortet (EK Votum 2162/2015) und entspricht der aktuellen Fassung der Deklaration von Helsinki. Alle Teilnehmer*innen wurden mittels ausführlicher Information über die Studie informiert und gebeten, eine Einverständniserklärung zu unterschreiben.

Im Rahmen dieser Querschnittserhebung wurden insgesamt 14 halbstrukturierte, leitfadengestützte Tiefeninterviews (siehe Online-Anhang) durchgeführt. Die Interviews fanden im Zeitraum 2018-2019 statt. Alle Teilnehmer*innen wurden ausführlich über die Studie informiert und gebeten, vor der Teilnahme an der Studie eine detaillierte Informations-/Einverständniserklärung zu unterschreiben. In den Tiefeninterviews wurde mit offenen Fragen nach den Faktoren gefragt, die für die Entscheidung für oder gegen eine Psychiatrieausbildung ausschlaggebend waren oder sind. Diese Interviews wurden von den 4 Autor*innen - 1 Fachärztin für Psychiatrie und 3 Assistenzärzt*innen für Psychiatrie - persönlich an der Universitätsklinik für Psychiatrie und Psychotherapie der Medizinischen Universität Wien durchgeführt und dauerten zwischen $30 \mathrm{Mi}$ nuten und 1 Stunde. Alle Interviews wurden mit Audiorecordern aufgezeichnet und wortwörtlich transkribiert.

\section{Studienteilnehmer*innen}

Im Rahmen der Studie wurde die Gruppe der Interviewten aus Personen zu unterschiedlichen Zeitpunkten ihrer Ausbildung und aus unterschiedlichen beruflichen Einsatzbereichen (Ambulanz/ Station) zusammengesetzt, um ein unterschiedliches Ausmaß der Fachzugehörigkeit zu gewährleisten. Es wurden 3 Medizinstudent*innen aus Österreich und Deutschland sowie 7 Ärzt*innen in Ausbildung für Psychiatrie und 4 Fachärzt*innen für Psychiatrie, die in verschiedenen Kliniken und Ambulanzen in Wien arbeiteten, befragt. Da ein Drittel der Studienbewerber*innen für das Medizinstudium in Österreich aus Deutschland stammt, wurde auch ein deutscher Medizinstudent interviewt [14].

\section{Interviewleitfaden und Auswertung}

Basierend auf einer Literaturrecherche zu diesem Thema wurden offene Fragen für ein halbstrukturiertes Interview formuliert, die Einstellungen und Interessen zur Psychiatrie umfassten. Halbstrukturierte Interviews gewährleisten Flexibilität, um auf individuelle Themen und Fragen einzugehen, wenn sie auftauchen, und gleichzeitig alle Interessenbereiche abzudecken. Die Fragen waren offen und wurden je nach Bedarf überarbei- 
tet, um ein breites Spektrum an unterschiedlichen Themen anzusprechen.

Die Transkripte der Interviews wurden analysiert und nach einem induktiven Codierungsschema codiert. Die Transkripte wurden von mindestens 2 Forscherinnen bzw. Autorinnen wobei eine Person umfassende Erfahrung in qualitativer Forschung aufweisen konnte, eine weitere moderate Erfahrung gelesen, um Themen von Interesse zu identifizieren. Drei der 4 Autorinnen waren zum Zeitpunkt der Studie in Ausbildung zu Fachärztinnen für Psychiatrie, wobei 2 der 3 bereits weit fortgeschritten in ihrer Ausbildung waren, 1 Autorin war Fachärztin für Psychiatrie. Die Themen von Interesse wurden von jeder Forscherin codiert. Der nächste Schritt bestand darin, die Codierungen der Forscherinnen zu vergleichen und zu diskutieren, ob ähnliche Interessengebiete oder Themen und Muster vorhanden waren. Es wurde ein Codierungsrahmen entwickelt. Es handelt sich um ein konsensuelles Codierungsverfahren, da die Kategorien nur nach gegenseitiger Übereinstimmung festgelegt wurden. Der Codierungsrahmen wurde auf Relevanz und Konsistenz überprüft und bei Bedarf modifiziert. Die Transkripte wurden wiederholt codiert und mit dem überarbeiteten Codierungsrahmen verglichen. Wichtige Themen wurden in die nachfolgenden Interviews aufgenommen, bis eine theoretische Sättigung erreicht war. Die Auswertung erfolgte nicht nach jedem einzelnen Interview, sondern in Forschungstreffen während des Interviewprozesses wurden auftauchende Themen thematisiert und in die Folgeinterviews übernommen. Dieser Prozess führte zum Auftauchen relevanter Inhalte, die dann in verschiedene Kategorien eingeordnet wurden.

\section{Ergebnisse}

\section{Soziodemografische Merkmale der Teilnehmer*innen}

Zum Interviewzeitpunkt betrug das Alter der Teilnehmer*innen zwischen 24 und 70 Jahren. Der Altersdurchschnitt lag bei 37,8 Jahren. Die Mehrzahl der Befragten war männlich ( $n=9 ; 64,3 \%)$. Drei Teilnehmer*innen waren Medizinstudent*innen (21,4\%), 7 Teilnehmer*innen Assistenzärzt*innen (50\%) und 4 Teilnehmer*innen Fachärzt*innen (28,6\%). Die Mehrheit arbeitete im stationären Bereich ( $n=12 ; 85,7 \%) 2$ Teilnehmer*innen im ambulanten Bereich ( $n=2 ; 14,3 \%)$. Die Medizinstudent*innen wurden auch unter „stationär“ angeführt, absolvierten jedoch lediglich vorübergehend ein Praktikum (ฉ Tab. 1).

\section{Ergebnisse nach induktiven Codierungsschema ( Tab. 2)}

Kategorie I: Positive Aspekte des Fachs

Die zwischenmenschliche Ebene wurde von den Befragten als einer der Hauptunterschiede im Vergleich zu anderen Fächern hervorgehoben. Das Ausmaß des Kontakts, die Intensität oder Länge der Gespräche mit den Patient*innen wurde mehrfach betont (I1MedStud) (I2MedStud), ebenso die Bedeutung der Sprache als zentrales Kommunikationsmittel, als therapeutisches Werkzeug (17MedStud). „Die Psychiatrie nimmt sich Zeit. Die Psychiatrie hat den Anspruch, dass man Zeit braucht für die Patient ${ }^{*}$ innen, verglichen mit anderen Fächern, die oft mit der Hek-
- Tab. 1 Darstellung der Teilnehmer*innen mit Nummerierung, Ausbildung, Geschlecht, Alter und Arbeitsbereich (stationär/ambulant).

\begin{tabular}{|c|c|c|c|c|}
\hline $\begin{array}{l}\text { Teilneh- } \\
\text { mer*innen } \\
\text { Nummer }\end{array}$ & $\begin{array}{l}\text { Ausbil- } \\
\text { dung }\end{array}$ & $\begin{array}{l}\text { Ge- } \\
\text { schlecht }\end{array}$ & Alter & $\begin{array}{l}\text { Arbeits- } \\
\text { bereich }\end{array}$ \\
\hline 1 & $\begin{array}{l}\text { Medizin- } \\
\text { student }\end{array}$ & $\mathrm{m}$ & 24 & stationär \\
\hline 2 & $\begin{array}{l}\text { Medizin- } \\
\text { studentin }\end{array}$ & w & 25 & stationär \\
\hline 3 & $\begin{array}{l}\text { Assistenz- } \\
\text { ärztin }\end{array}$ & w & 33 & stationär \\
\hline 4 & Facharzt & $\mathrm{m}$ & 70 & ambulant \\
\hline 5 & Facharzt & $\mathrm{m}$ & 56 & ambulant \\
\hline 6 & Fachärztin & w & 62 & stationär \\
\hline 7 & $\begin{array}{l}\text { Medizin- } \\
\text { student }\end{array}$ & $\mathrm{m}$ & 30 & stationär \\
\hline 8 & $\begin{array}{l}\text { Assistenz- } \\
\text { arzt }\end{array}$ & $\mathrm{m}$ & 33 & stationär \\
\hline 9 & Facharzt & $\mathrm{m}$ & 39 & stationär \\
\hline 10 & $\begin{array}{l}\text { Assistenz- } \\
\text { ärztin }\end{array}$ & w & 32 & stationär \\
\hline 11 & $\begin{array}{l}\text { Assistenz- } \\
\text { arzt }\end{array}$ & $\mathrm{m}$ & 33 & stationär \\
\hline 12 & $\begin{array}{l}\text { Assistenz- } \\
\text { arzt }\end{array}$ & $\mathrm{m}$ & 29 & stationär \\
\hline 13 & $\begin{array}{l}\text { Assistent- } \\
\text { ärztin }\end{array}$ & w & 31 & stationär \\
\hline 14 & $\begin{array}{l}\text { Assistenz- } \\
\text { arzt }\end{array}$ & $\mathrm{m}$ & 32 & stationär \\
\hline
\end{tabular}

tik des Krankenhausalltags zu kämpfen haben" (I10Ass). Auch der Aufbau von Beziehung, das Zuhören und die partnerschaftliche Zusammenarbeit mit den Patient*innen wurden positiv hervorgehoben (I7MedStud). „Die Nähe zu den Patient*innen und die Kommunikation mit den Menschen" (I12Ass) (I9FA) wurde von den Interviewteilnehmer* innen besonders betont. Die Psychiatrie geht mit einem „sehr positiven Menschenbild an die Sache heran (...) (und nimmt) Ressourcen (von Patient*innen) wahr (...)“ (I12Ass).

Ein weiterer Aspekt war der ganzheitlich-interdisziplinäre Ansatz der Psychiatrie, welcher den Menschen als Ganzes betrachtet und ihn in den Mittelpunkt stellt (I1MedStud) (I7MedStud) (I13Ass) (I14Ass) (I4FA) (I5FA) (I9FA). Die Psyche ist umfassender als einzelne Körperteile und das Gehirn fungiert als Zentrum der Organe und des Bewusstseins (I8Ass) (I4FA). „(...) weil ja die Psyche Teil des Menschen ist und man/frau viel zu sehr im Laufe der Jahre sich immer nur auf die somatischen Sachen, also die Organbeschwerden reduziert hat und immer mehr auch auf technologische Entwicklungen (...) und die Psyche aus den anderen Fächern immer mehr verschwunden ist" (I2MedStud). Das Fach erfordert multidimensionales sowie multifaktorielles und multikausales Denken (I14Ass). Die Psychiatrie versucht objektiv 
- Tab. 2 Darstellung der 9 Hauptthemen, eingeteilt in 3 Kategorien.

Kategorie I: Positive Aspekte des Fachs

die zwischenmenschliche Ebene

z. B. die umfassende Kommunikation mit den Patient* innen (I12Ass; I9FA)

das Ganzheitliche/Interdiszipli-

z. B. Multiprofessionalität im

näre Team (I10Ass; I2MedStud)

das Bild der Psychiater*innen z. B. Fertigkeiten wie Zuhören können (I10Ass)

das Bild der psychiatrischen

Patient*innen

z. B. kreative und starke Menschen (I5FA)

Kategorie II: Negative Aspekte des Fachs

fehlende Exaktheit in Diagnostik und Behandlung

z. B. Diagnostik beruht auf Konventionen und Erfahrung und nicht auf biologischen Grundlagen (I4FA; I10Ass)

anstrengende Arbeit/negatives Bild über psychiatrische Paz. B. Aspekte des Zwanges in der tient*innen Psychiatrie (I12Ass; I14Ass).

Stigma/Image unter Kolleg*innen

z. B. das Stigma innerhalb der Medizin (I10Ass; I2MedStud)

Kategorie III: Motivatoren zur Fachwahl

Kennenlernen von psychisch Erkrankten

z. B. während des Zivildienstes in psychiatrischen Einrichtungen oder im Studium (I5FA).

Gestaltung der Lehre z. B. gute Supervision im klinisch praktischen Jahr (I1MedStud) terviewter. Diejenigen, die in diesem Beruf arbeiten, erfüllen die Anforderungen des Fachs wie Kommunikationsfähigkeit, Arbeiten im Team und Fertigkeiten wie Zuhören (I10Ass). Psychiater*innen sollten gut strukturiert sein und gut sprechen können, sie sollten sehr empathisch sein (I8Ass) (I10Ass). „Positiv ist, dass man während der Ausbildung sehr wachsen kann im Sinne einer Persönlichkeitsentwicklung“ (I10Ass). Das Fach bietet einen breiten Zugang zum Menschen, die Ausbildung in psychotherapeutischer Medizin verpflichtet zur Selbsterfahrung und Supervision, dadurch lernt man zu reflektieren und im besten Fall ist auch eine persönliche Weiterentwicklung möglich (I1MedStud) (I10Ass). „(...) dass es eine gute Übung ist, für das Menschsein an sich, dieses Psychiater*in sein" (I12Ass). Die Psychiater*innen wären darüber hinaus "auch oft künstlerisch“ weil Kreativität gefragt ist, wenn sie individuelle Lösungen für Patient*innen suchen (I11Ass) (I13Ass).

Das Bild von psychiatrischen Patient*innen als kreative, spannende, vielfältige und starke Menschen (I5FA), die sich Widrigkeiten stellen und immer wieder standzuhalten versuchen, besteht (I11Ass). Sätze wie „Ich kann mindestens genauso viel von denen lernen, wie die hoffentlich von mir" (I11Ass) und „(...) dass die Patient ${ }^{*}$ innen meist auch deutlich motivierter sind (...) und offener sind für Behandlungsideen und Konzepte" (I9FA) wurden dabei angemerkt. Schließlich wurde auch betont, dass es kein stereotypes Bild von psychiatrischen Patient*innen gibt, das auf alle passen würde (I5FA). Ein spannender Aspekt ist der Krankheitsbegriff (I5FA) bzw. die Grenzen zwischen gesund und krank bzw. "normal“ und "nicht mehr normal“ (I12Ass) und dem Graubereich sowie die Berücksichtigung der psychosozialen Aspekte von Erkrankungen (I10Ass).

\section{Kategorie II: Negative Aspekte des Fachs}

Die Angst vor dem Unbekannten in der Psychiatrie, ist auch etwas, das in der Öffentlichkeit vorherrscht (I1MedStud) (I9FA) und die dadurch bestehende Hemmschwelle überhaupt Behandlung zu suchen (I2MedStud).

Die fehlende Exaktheit in Diagnostik und Behandlung (I4FA) (I10Ass) sowie die bestehenden Forschungslücken im Bereich der biologisch-organisch orientierten Forschung, die hinter anderen medizinischen Fächern zurückbleibt, zeigen Defizite (I4FA) (I5FA). Die Psychiatrie kann viele Störungen gut beschreiben, aber deren Ursache oft nicht hinreichend erklären (I8Ass). Schwierigkeiten in der Diagnose ergeben sich auch, wenn „Menschen nur im Querschnitt“ gesehen werden (I9FA). In der Diagnostik gäbe es zu wenig evidenzbasierte Vorgehensweisen. Andere Fächer verwenden objektivere Kriterien, die durch Labor und Radiologie quantitativ gemessen werden können (I2MedStud) (I10Ass) (I13Ass). Der Großteil der psychiatrischen Diagnostik beruht auf Konventionen und Erfahrung und nicht auf biologischen Grundlagen (I4FA) (I10Ass). Es geht um die psychopathologische Beurteilung und die Einschätzung von Suizidalität. Psychiatrische Störungen werden oftmals in der Gesellschaft nicht als Krankheiten wahrgenommen. Die Vorurteile über Psychopharmaka als Medikamente zur Ruhigstellung und „potenziell persönlichkeitsverändernde Medikamente" (17MedStud) wurden angesprochen. Auch Nebenwirkungen von Psychopharmaka, Polypharmazie und Wechselwir- 
kungen sind ein Problem (I11Ass) (I13Ass). „Und dass auch die Behandlungen teilweise nicht so effektiv sind wie in anderen Fachbereichen und dass es auch sehr lange Behandlungszeiten sind an sich“ (I2MedStud) und „Interventionsmöglichkeiten sehr viel begrenzter sind, als in operativen oder internistischen Fächern “ (I9FA).

Ein weiterer negativer Aspekt sind zahlreiche Vereinsgründungen von Psychotherapieschulen in Österreich (I14Ass) (I6FÄ), die polarisierend wirken. Die unzureichende Finanzierung von Psychotherapie durch die Krankenkassen (I4FA) und eine zu schwache politische Interessenvertretung wurden thematisiert (I10Ass).

Die anstrengende Arbeit mit Patient*innen und die Aspekte des „Zwang(es) in der Psychiatrie“ wurden als besondere Belastung betont (I12Ass) (I14Ass). Vor allem die Arbeit mit schwer kranken untergebrachten Patient*innen, mit denen man einerseits eine Beziehung aufbauen müsse, um sie behandeln zu können, aber genug Distanz herstellen müsse um klare Grenzen zu schaffen, (17MedStud) (I10Ass) wurden genannt. Um Missbrauch und Ausnutzung von Machtverhältnissen vorzubeugen (I6FÄ) (I12Ass), brauche man mehr Zeit, um den Kontakt zu Patient*innen zu reflektieren (I10Ass). Als negatives Beispiel aus der Geschichte wurde die intellektuelle und strukturelle Verwicklung der Psychiatrie in Verbrechen des Nationalsozialismus erwähnt (I1MedStud) (I12Ass).

Ein negatives Bild von psychiatrischen Patient*innen herrsche außerhalb der Psychiatrie vor „(...) es ist natürlich einfach ein psychiatrischer Patient bzw. eine Patientin, dieser wird sehr oft einfach von allen anderen abgeschoben und der ist ja eh nur verrückt“ (I2MedStud). Psychiatrische Patient*innen werden oft wahrgenommen als würden sie „ein System stören“ oder „nicht hineinpassen“ (I12Ass). Auch bei Medizinstudent*innen bestehe ein negatives Image der Psychiatrie „In der Psychiatrie sind die Depperten“ (I14Ass), welches jedoch ein gesellschaftliches Problem einer negativen Wahrnehmung von Patient*innen widerspiegle. Medizinstudent*innen kommen mit einem gewissen Bild geprägt durch die Herkunftsfamilie oder den Bekanntenkreis an die Universität (I14Ass). Nicht die Psychiatrie als Einrichtung ist verantwortlich für ihren schlechten Ruf, sondern die Begegnung der Menschen mit psychiatrischen Erkrankungen.

Das Stigma, das dem Fach anhaftet innerhalb der Medizin (I10Ass) (I2 MedStud), die fehlende Attraktivität durch schlechtere Verdienstmöglichkeiten verglichen mit anderen Fächern (I12Ass) (I14Ass), sowie das schlechte Image unter Kolleg*innen sind nur einige negative Aspekte (I14Ass). Oberärzt*innen bzw. Lehrende, die sich negativ über Psychiater*innen äußern, tragen zu einer fehlenden Wertschätzung bei (I7MedStud) (I10Ass). Vorurteile wie, dass viele Psychiater*innen dieses Fach aufgrund von Interesse an eigenen psychiatrischen Schwierigkeiten wählen würden, wurden diskutiert (I4FA). Von Familienangehörigen und Bekannten oder Mitstudierenden (I7MedStud) (I9FA) hört man „Du willst Psychiatrie machen? Willst du nicht lieber (...) richtige Medizin machen?" (I1MedStud) oder Kommentare von Kolleg*innen aus anderen Fächern wie „du bist doch zu intelligent darfür, verschwende dies nicht“ (I2MedStud). Das Losgelöstsein der Psychiatrie von anderen Fächern der Medizin wurde als weiteres Beispiel für das Stigma genannt (I6FÄ). Dabei wurde und ist „die Psychiatrie architekto- nisch exkludiert und verbannt. Weil die Psychiatrie ein eigenes Gebäude hat und alle anderen sind drüben. Das ist auch schon eine deutliche Trennung“ (I2MedStud).

\section{Kategorie III: Motivation zur Fachwahl}

Ein gewisses grundlegendes Interesse für Menschen und ein ganzheitlicher Blick würden die Wahl dieses Faches begünstigen (I11Ass). Die Möglichkeit, viel über zwischenmenschliche Beziehungen zu lernen, ein positives Arbeitsklima und gute Arbeitsbedingungen, weniger Ellbogentechnik in der Psychiatrie, erscheinen attraktiv für den Karriereweg (I2MedStud) (I7MedStud). Altruistische Einstellungen wurden häufig als persönliche Motive genannt. Die vielleicht eigene etwas schwierige Lebenserfahrung könnte ebenfalls als Motivator dieses Fach zu wählen dienen aber auch Vorbilder in der Familie (z. B. Vater als Neurologe - eine andere Art Psychiater) (I4FA) (I11Ass). Die Auseinandersetzung mit psychotherapeutisch orientierten Inhalten in der Maturaklasse im Fach Philosophie und Psychologie bereitete in der schulischen Laufbahn den Weg (I5FA).

Das Kennenlernen von psychisch leidenden Menschen und der konkrete Kontakt mit ihnen wurde im Rahmen des Zivildienstes in psychiatrischen Einrichtungen oder als Betreuer in Wohngemeinschaften während des Studiums als Motivator genannt (I5FA).

Das bestehende akademische Interesse am „psychischen Apparat" und auch an psychodynamischen Konzepten motivierten ebenfalls dazu die Fachärzt*innenausbildung zu wählen.

Gute Erfahrungen im Rahmen der Ausbildung (I1MedStud) (I2MedStud) (I7MedStud) (I12Ass) (I13Ass) inklusive psychiatrische Praktika, Tertial und dem Klinisch Praktischen Jahr, eine gute Diplomarbeitsbetreuung und gute Supervision als Student trugen ebenfalls dazu bei, die Angst vor dem Fach zu verlieren (I1MedStud) und sich in Inhalte zu vertiefen (I10Ass). Ein Interviewteilnehmer kommentierte seine wegweisenden Erfahrungen im Studienpraktikum mit „(...) was mich besonders beeindruckt hat war sozusagen diese Menschlichkeit und diese Wertschätzung, die den Menschen mit psychiatrischen Erkrankungen gegeben wurde und das war sehr eindrucksvoll für mich“ (I9FA). Lehrende, die das Bild der Psychiatrie positiv beeinflusst haben (I2MedStud) und die die Gesprächsführung gut beherrscht haben (I10Ass) sowie besondere Vorbilder der akademischen Psychiatrie (I6FÄ), wurden genannt.

Das sich entwickelnde Interesse an Psychosomatik, nachdem die Ausbildung zum Allgemeinmediziner absolviert wurde (I6FÄ), oder die pragmatische Argumentation etwa, dass Psychiatrie körperlich weniger anstrengend wäre, als z. B. die Chirurgie, fanden Erwähnung (I4FA).

Als Anregung, um mehr Medizinstudent*innen zu motivieren Psychiatrie als Karrierefach zu wählen, wurde eine bessere Gestaltung der Lehre erwähnt. Eine individuelle Betreuung der Studierenden wäre wünschenswert. Die Psychiatrie sollte nicht isoliert als Einzelfach stehen -, sondern psychiatrische Aspekte sollten in den Unterricht anderer Fächer, z. B. gynäkologische Psychosomatik, integriert werden. „In jedem Fach der Medizin gäbe es eigentlich ein bisschen Psychiatrie“ (I10Ass). Der konkrete Kontakt zu psychisch Kranken bereits im Studium, um diese Menschen kennenzulernen, wäre ausschlaggebend und sollte 
intensiver gefördert werden. Die Fachärzt*innenausbildung sollte nicht ausschließlich im Krankenhaus stattfinden, sondern auch im niedergelassenen Bereich (I1MedStud). Darüber hinaus bieten gute Jobaussichten als Mangelfach und die mögliche Tätigkeit in verschiedenen Settings (z. B. eigenständig in einer Ordination, als Teammitglied im stationären oder ambulanten Bereich oder in einer Tagesklink) Anreize für eine Fachärzt*innenausbildung im Fach Psychiatrie (I12Ass).

\section{Diskussion}

Die Studie ist innovativ, da in bisher keiner (den Autorinnen bekannte) Studie zu diesem Thema qualitative Methoden zur Anwendung kamen. Die Ergebnisse ermöglichen weitere wichtige Schritte, um das Interesse von Medizinstudent*innen als zukünftige Fachärzt*innen für Psychiatrie langfristig zu sichern, indem die Erfahrungen und Ideen von bestehenden Fachärzt*innen und Ausbildungskandidat*innen in mögliche geplante Maßnahmen einbezogen werden können.

Einige der in den Ergebnissen erwähnten Punkte wurden bereits in der Vergangenheit gefunden. Der Einfluss des Stigmas und der Vorurteile gegenüber der Psychiatrie sowie die Verbreitung durch das System selbst (Dozent*innen, andere Student*innen) beeinflussen das Interesse der Student*innen. Eine der neueren Veröffentlichungen zu diesem Thema, eine Online-Querschnittsstudie mit 502 Medizinstudent*innen, kam zu dem Schluss, dass die Studierenden die Psychiatrie zwar aufgrund negativer Einstellungen gegenüber dem Fach ablehnten, nicht aber aufgrund der Stigmatisierung psychisch Kranker [15]. In dieser Studie wurde auch der Einfluss der Erwartungen, von Familie und Freunden hervorgehoben. Der Einfluss der Meinung von Angehörigen und Freunden wurde auch in unserer Studie als Ergebnis repliziert.

Ein weiterer wichtiger Punkt war die Erfahrung mit eigenen psychischen Schwierigkeiten als Motivation für eine Ausbildung in der Psychiatrie. Innere Konflikte oder erhöhte Ängste, wurden bereits in einer früheren Studie angegeben [16]. Eagle et al. fanden, dass Medizinstudent*innen, die alleinstehend waren, aus größeren Städten stammten, wenig Interesse an Politik und größeres Interesse an humanitären Ideen bzw. Menschenrechten hatten, wenig Affinität zu autoritären Ideen und die eher bereit waren, Mehrdeutigkeiten auszuhalten, eher dazu neigten, Psychiatrie als Fach zu wählen. Auch die Bereitschaft, Verantwortung für Patient*innen zu übernehmen, korrelierte positiv mit der Fächerwahl Psychiatrie [17].

Erwähnenswert ist, dass die Entscheidung Psychiatrie als Karrierefach zu wählen, mehrheitlich während der Studienzeit getroffen wurde, wie eine retrospektive amerikanische Studie an über 29000 Medizinstudent*innen zeigte [18]. Jene Student*innen die bereits bei Studienbeginn Psychiatrie gewählt hatten, blieben mehrheitlich bei ihrer Wahl.

Geplante Interventionen sollten im Zeitraum vor dem Studium und während des Studiums gesetzt werden, um die Rekrutierung in das Fach zu erhöhen. Die Gestaltung der Lehre scheint ein wesentlicher Faktor zu sein, der die Berufswahl beeinflusst. Die Integration von psychiatrischen Aspekten in patient* innenbezogenen Falldarstellungen wäre ein wichtiger As- pekt, der von Interviewteilnehmer*innen wiederholt genannt wurde. Auch die Qualität der Lehre wurde mehrfach erwähnt. Ein 8-wöchiges psychiatrisches Famulaturpraktikum an der Medical University of Western Australia zeigte nur mäßige Auswirkungen auf bestehende Einstellungen, Vorurteile und Überlegungen zur Berufswahl der Studierenden [19]. Um die Rekrutierung von Medizinstudent*innen für die Psychiatrie zu verbessern, organisierte die psychiatrische Abteilung an der University of Western Australia seit 2009 eine jährliche „Schnupperwoche“, in der Student*innen die Möglichkeit hatten, eine Reihe von interaktiven Seminaren zu psychiatrischen Themen zu besuchen sowie mit Psychiater*innen und solchen in der Ausbildung zu sprechen [20].

Ein Vorschlag der Interviewteilnehmer*innen der vorliegenden Studie, um mehr Studierende für das Fach Psychiatrie zu gewinnen, war die Integration von Strategien zur Stigmaüberwindung (I6FÄ) bereits in der vorklinischen Lehre. Der Schlüssel zur Überwindung von Stigma ist eine Einführung in das Fach Psychiatrie, die es den Studierenden ermöglicht, psychisch Kranke persönlich kennenzulernen. Das persönliche Gespräch zwischen Medizinstudierenden und Patient*innen wird an der Medizinischen Universität Wien bereits eingesetzt, allerdings erst im Tertialpraktikum, gegen Ende des Studiums wo die Studierenden 3 Fallvorstellungen präsentieren müssen. Das persönliche Gespräch mit psychisch Kranken führt zu einer verbesserten Einstellung gegenüber dem Fach Psychiatrie. Ähnliche Erkenntnisse brachte eine kürzlich publizierte Untersuchung an 40 Medizinstudent*innen, welche im Rahmen eines speziellen Programms Erfahrungen in der Psychiatrie sammeln durften. Die Ergebnisse zeigten deutliche Verbesserung der Einstellung nach persönlichem Kennenlernen von psychiatrisch Erkrankten [21]. Vergleichbare Ergebnisse zeigte eine breit angelegte Online-Studie aus Deutschland, Schweiz, Österreich und Ungarn an über 1300 Medizinstudent*innen, welche ebenfalls die persönliche Erfahrung sowie die curriculumbasierte Erfahrung mit Psychiatrie als wichtigste beeinflussende Faktoren darstellte [22].

Die häufig vorhandene Hemmschwelle kann durch den persönlichen Kontakt überwunden werden und die Student*innen machen die Erfahrung, dass das Gespräch mit psychisch Kranken meist ganz anders verläuft als sie es sich möglicherweise vorstellen. Durch die reale Erfahrung vergehen Ängste oder überzogene Erwartungen.

Das Stigma, das der Psychiatrie anhaftet, müsste überwunden werden durch Awareness-Kampagnen in der breiten Öffentlichkeit. Die Verbesserung des Stellenwerts der Psychiatrie in der Gesellschaft kann in erster Linie durch eine Aufwertung innerhalb des medizinischen Systems selbst stattfinden.

Es muss Vorbilder geben, die den Medizinstudent*innen zeigen wie es funktioniert mit den Patient*innen zu kommunizieren und zuzuhören. Das geht nur durch persönlichen Kontakt mit den Studierenden.

Eine Intensivierung der Praktika wäre notwendig, denn durch persönliche Erfahrung entsteht Interesse am Fach und das Bild, das man sich über Psychiatrie macht, kann stark geprägt und verändert werden. Ein Erlernen und Verbesserung der ärztlichen Gesprächsführung, kann nicht nur eine große 
Hemmschwelle für Medizinstudent*innen darstellen, sondern betrifft auch Kolleg*innen aus anderen Fächern, die sagen „ich könnte das gar nicht, die ganze Zeit mit meinen Patient*innen reden“. Eine bessere Lehre an Abteilungen, die ein Interesse bei den Student*innen anregt und sie nicht mit einer hohen Bringund Holschuld zurücklässt, wäre wünschenswert.

\section{Stärken und Schwächen}

Eine Schwäche der Studie ist sicherlich die Befangenheit der Autor*innen, die Interesse haben, das eigene Fach positiver darzustellen, um mögliche Motivationsfaktoren für Psychiatrie als Karriereweg zu identifizieren. Eine weitere Schwäche der Studie ist die unausgewogene Zusammensetzung des Untersuchungssamples, in dem Ärzt*innen in Ausbildung für Psychiatrie im Verhältnis zu den Medizinstudent*innen und Fachärzt*innen überrepräsentiert sind. Die Stärken der Studie liegen in der Relevanz der Thematik in Hinblick auf den fehlenden Nachwuchs eines Mangelfachs sowie die qualitative Forschungsmethodik mit einem breiteren Ansatz, welcher Aspekte berücksichtigt, die in bisherigen Fragebogenuntersuchungen zu kurz kamen. Die Studie kann als Grundlage für weitere tiefergehende Arbeiten genutzt werden und erfüllt damit einen wichtigen Zweck.

\section{KONSEQUENZEN FÜR KLINIK UND PRAXIS}

- Der steigende Bedarf an psychiatrischer Versorgung in Österreich sieht eine hohe Anzahl von Fachärzt*innen für Psychiatrie vor, zu wenig Medizinstudent*innen wollen Psychiater*innen werden.

- Das vorhandene Stigma um das Fach wird durch manche Vertreter*innen des medizinischen Systems selbst aufrechterhalten.

- Aus Sicht von Fachärzt*innen der Psychiatrie, Ärzt*innen in Fachärzt*innenausbildung und Medizinstudent*innen zeigten sich im Rahmen der qualitativen Erhebung Berührungsängste als einer der Hauptgründe.

- Ein möglichst frühes persönliches Kennenlernen von psychiatrischen Patient*innen im Rahmen des Medizinstudiums verbessert Einstellungen.

\section{Interessenkonflikt}

Die Autorinnen/Autoren arbeiten in dem Fachgebiet, das sie untersuchen.

Literatur

[1] Kretschmer F. Alarmstufe in der Psychiatrie. 30.05.2014: http://derstandard.at/2000001665297/Alarmstufe-in-der-Psychiatrie; Stand: 30.03.2021

[2] Kalma MRCR. Abschätzung des zukünftigen Bedarfs an Psychiatern in Österreich. Studie im Auftrag und in Kooperation mit der Österreichischen Gesellschaft für Neuropsychopharmakologie und Biologischen Psychiatrie (ÖGPB). 2011: https://irihs.ihs.ac.at/id/eprint/ $2100 /$
[3] Lyons Z. Attitudes of Medical Students Toward Psychiatry and Psychiatry as a Career: A Systematic Review. Acad Psychiatry 2013; 37: 150. doi:10.1176/appi.ap. 11110204

[4] Andlauer O, Van Effenterre A, Haffen E et al. Encouraging French medical students to choose a career in psychiatry: How and why? Int Rev Psychiatry 2013; 25: 460-465. doi:10.3109/09540261.2013.821404

[5] Pailhez G. Attitudes and Views on Psychiatry: A Comparison Between Spanish and U.S. Medical Students. Acad Psychiatry 2005; 29: 82-91. doi:10.1176/appi.ap.29.1.82

[6] Aslam M, Taj T, Ali A et al. Psychiatry as a career: a survey of factors affecting students' interest in psychiatry as a career. Mcgill J Med 2009; 12: 7-12. doi:10.26443/mjm.v12i1.730

[7] Katschnig H. Are psychiatrists an endangered species? Observations on internal and external challenges to the profession. World Psychiatry 2010; 9: 21-28. doi:10.1002/j.2051-5545.2010.tb00257.x

[8] Silverman L. Texas Strives to Lure Mental Health Providers to Rural Countries. 03.09.2015: https://khn.org/news/texas-strives-to-luremental-health-providers-to-rural-counties/; Stand: 30.03.2021

[9] Desai R, Panchal B, Vala A et al. Impact of clinical posting in psychiatry on the attitudes towards psychiatry and mental illness in undergraduate medical students. Gen Psychiatry 2019; 32: e100072. doi:10.1136/gpsych-2019-100072

[10] Burra P, Kalin R, Leichner P et al. The ATP 30-a scale for measuring medical students' attitudes to psychiatry. Med Educ 1982; 16: 31-38. doi:10.1111/j.1365-2923.1982.tb01216.x

[11] Shankar R, Laugharne R, Pritchard C et al. Modified Attitudes to Psychiatry Scale Created Using Principal-Components Analysis. Acad Psychiatry 2011: 35. doi:10.1176/appi.ap.35.6.360

[12] Stuart H, Sartorius N, Liinamaa T. Images of psychiatry and psychiatrists. Acta Psychiatr Scand 2015; 131: 21-28. doi:10.1111/ acps. 12368

[13] Kassam A, Glozier N, Leese M et al. Development and responsiveness of a scale to measure clinicians' attitudes to people with mental illness (medical student version). Acta Psychiatr Scand 2010; 122: 153161. doi:10.1111/j.1600-0447.2010.01562.x

[14] Schlager R. Vier von zehn Medizinbewerbern sind Deutsche. Die Press; 15.04.2017: https://www.diepresse.com/5201507/vier-vonzehn-medizinbewerbern-sind-deutsche; Stand: 30.03.2021

[15] Seow LSE, Chua BY, Mahendran R et al. Psychiatry as a career choice among medical students: a cross-sectional study examining schoolrelated and non-school factors. BMJ Open 2018; 8: e022201. doi:10.1136/bmjopen-2018-022201

[16] Maron BA, Fein S, Maron BJ et al. Ability of Prospective Assessment of Personality Profiles to Predict the Practice Specialty of Medical Students. Baylor Univ Med Cent Proc 2007; 20: 22-26. doi:10.1080/ 08998280.2007 .11928228

[17] Eagle P, Marcos L. Factors in medical students' choice of psychiatry. Am J Psychiatry 1980; 137: 423-427. doi:10.1176/ajp.137.4.423

[18] Goldenberg MN, Williams DK, Spollen JJ. Stability of and Factors Related to Medical Student Specialty Choice of Psychiatry. Am J Psychiatry 2017; 174: 859-866. doi:10.1176/appi.ajp.2017.17020159

[19] Lyons Z, Janca A. Impact of a psychiatry clerkship on stigma, attitudes towards psychiatry, and psychiatry as a career choice. BMC Med Educ 2015; 15: 34. doi:10.1186/s12909-015-0307-4

[20] Lyons Z, Power B, Bilyk N et al. The University of Western Australia Institute of Psychiatry for Medical Students: An Australian First. Australas Psychiatry 2009; 17: 306-310. doi:10.1080/10398560902964602

[21] Holt C, Mirvis R, Bao J et al. Three-Year Longitudinal Follow-up of the Psychiatry Early Experience Program (PEEP): Gaining and Sustaining Positive Attitudes Towards Psychiatry in Students at a UK Medical School. Acad Psychiatry 2019; 43: 600-604. doi:10.1007/s40596019-01092-0

[22] Warnke I, Gamma A, Buadze M et al. Predicting Medical Students' Current Attitudes Toward Psychiatry, Interest in Psychiatry, and Estimated Likelihood of Working in Psychiatry: A Cross-Sectional Study in Four European Countries. Front Psychiatry 2018; 9. doi:10.3389/ fpsyt.2018.00049/full 